\title{
Simulation dynamique d'un moteur : cas du Stirling de type gamma
}

\author{
N. Martaj $^{1}$, R. Bennacer ${ }^{2}$, P. Rochelle ${ }^{1}$ et L. Grosu ${ }^{1}$ \\ 1 Laboratoire d'Énergétique et d'Économie d'Énergie, 50 rue de Sèvres, 92410 Ville d'Avray, France \\ 2 Laboratoire LEEVAM, Univ. Cergy, 5 mail Gay Lussac, 95031 Neuville sur Oise, France
}

Reçu le 12 juin 2008

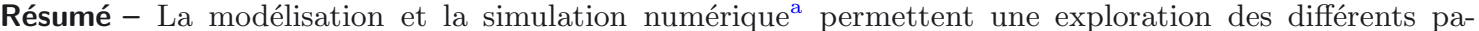
ramètres de contrôles afin d'identifier les conditions et géométries optimales de fonctionnement des systèmes. Cette approche permet entre autre d'obtenir les valeurs instantanées des grandeurs physiques locales. Ces outils numériques progressent et traitent des systèmes de plus en plus complexes confortés par la progression des puissances des ordinateurs. Nous décrirons dans le présent travail le cas de moteurs alternatifs de type Stirling, à combustion externe et régénération. Ces moteurs constituent une solution pour la conversion efficace des énergies renouvelables et des chaleurs perdues en travail. La simulation de tels systèmes en régime établi permettra d'en déduire les énergies échangées et de démontrer que ces moteurs offrent un bon rendement de fonctionnement, tout en présentant une grande souplesse d'adaptation. L'optimisation des machines est fortement liée à leurs paramètres géométriques et physiques (dimensions, matériaux, coefficient de transfert de chaleur, etc.) ce qui peut engendrer un coût important pour les différents prototypes. La simulation numérique permettra la prédiction du cycle au cours du temps et donnera accès aux valeurs du travail ainsi que le rendement de la machine simulée. Cette démarche identifiera les zones de fonctionnements optimaux et réduira le nombre de prototypes et du même coup le coût du projet. La modélisation s'appuie sur la résolution des équations de conservation d'écoulements compressibles anisothermes dans un moteur LTD en domaine bidimensionnel (2D, moteur supposé axisymétrique afin de faciliter l'illustration). En guise de validation l'un des résultats obtenus numériquement est confronté aux résultats expérimentaux obtenus sur un prototype moteur de démonstration. Le bon accord constaté sur le cycle $p-V$ illustre l'intérêt méthodologique.
\end{abstract}

Mots clés : Simulation numérique / moteur Stirling / conversion d'énergie

Abstract - Dynamic simulation of an engine: gamma-type Stirling case. Modelling and numerical simulation allow ${ }^{\mathrm{b}}$ the exploration of the different controlling parameters in order to identify the optimal working conditions. This numerical approach permits also to quantify instantaneous and local physical fields. These numerical tools are now resolving more and more complex systems with the help of the computer power increases. We describe in the present study the reciprocating thermal engine of Stirling type, with its varied external heat sources and efficiency improving regenerator. These engines are an alternate solution to be taken into account for an efficient conversion of renewable energies into work. For this use, a good operating efficiency and a great versatility are two qualities that distinguish this engine. Moreover, it is able of running at low temperature differential (LTD) which is especially attractive for low level heat recovery. The optimisation of such machine is strongly dependent of geometrical and physical parameters such as dimensions, heat transfer coefficients, heat source temperatures, regenerator characteristics. Such approach permits to get the instantaneous values of several local physical variables during established regime (pressure, temperature, speed and volume). The energy balance and the efficiencies are deduced.

${ }^{a}$ Réalisée dans la présente étude avec un maillage mobile (logiciel COMSOL).

b Using a multi-physics multi-D software. 
The approach is based on the numerical resolution of the compressible anisothermal fluid flow in an axisymetrical (2D) engine. As validation process, the obtained numerical results are compared with the available experimental results that we got on LTD Stirling engine prototype. The observed good agreement on the $P-V$ cycle illustrates the interest of the presented methodology.

Key words: Numerical simulation / Stirling engine / energy conversion

\section{Introduction}

Les outils numériques permettant la modélisation et la simulation progressent et traitent des systèmes de plus en plus complexes. Cette progression est le fruit d'un savoir-faire croissant d'une part et de l'accroissement de la puissance des ordinateurs d'autre part. Cette approche par l'utilisation de l'outil informatique permet une exploration à moindre coût des différents paramètres de contrôles afin d'identifier les conditions et géométries optimales de fonctionnement des systèmes. Cette approche permet entre autre d'obtenir les valeurs instantanées des grandeurs physiques locales dans des domaines très variés comme la croissance cristalline pour semiconducteurs $[1,2]$, la miniaturisation de l'électronique [3,4] etc.

Nous présentons comme application dans la présente étude le cas du moteur thermique Stirling. Ce moteur à « haut » rendement [5-7] permet d'obtenir un travail utile d'une conversion de chaleur à une température juste audessus ou au-dessous de la température ambiante. Par sa relative rusticité, son adaptabilité et ses propriétés thermodynamiques, il réduit les pertes par frottement ce qui est primordials au vu de la faible différence des températures.

Plusieurs variantes de moteur Stirling existent et on s'intéressera ici à l'une des configurations dite de type gamma (Fig. 1). La caractéristique de ce moteur réside dans les grandes dimensions des plaques d'extrémité du volume contenant le déplaceur qui sont utilisées comme échangeur de chaleur chaud ou froid.

Le diagramme indicateur théorique (Fig. 2), montre les quatre transformations subies par le gaz contenu dans le moteur. Afin d'accroître le rendement il est possible de réduire les pertes d'énergie (entre 3-4 et 1-2). Cette réduction est possible par l'ajout d'un régénérateur qui stocke l'énergie, par chaleur sensible, lors du passage du fluide du compartiment chaud vers le compartiment froid et de le restituer lors du passage inverse (entre 4-1 et 2-3). Le régénérateur est contenu dans le déplaceur, qui peut être poreux, ou bien, comme ici, dans l'espace annulaire compris entre le déplaceur et la paroi verticale du cylindre qui le contient.

\section{Modélisation et simulation}

L'état d'écoulement du fluide (ici de l'air) est décrit par le champ de vitesse, la masse volumique, la pression et la température. Ces variables sont à déterminer en chaque point de l'espace et à chaque instant à l'aide des équations de conservation de base suivantes :

- l'équation de continuité qui traduit la conservation de la masse de la particule fluide;
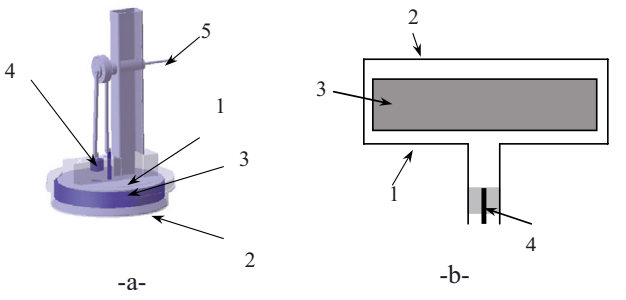

1. source froide,

2. source chaude,

3. piston déplaceur,

4. piston moteur,

5. arbre.

Fig. 1. Moteur Stirling de type gamma à faible différence de températures, (a) schéma du prototype, (b) schéma simplifié (2D).

- l'équation de la quantité de mouvement donnée par la seconde loi de Newton appliquée aux particules fluides ;

- l'équation de l'énergie qui exprime la conservation d'énergie de la particule fluide;

- l'équation d'état thermodynamique du fluide reliant les variables d'état $p, \rho$ et $T$.

La modélisation de cette transformation (écoulement et transfert) du gaz enfermé dans le cylindre moteur est présentée en annexe. Le principe de la démarche modélisation-simulation est schématisé ci-dessous :

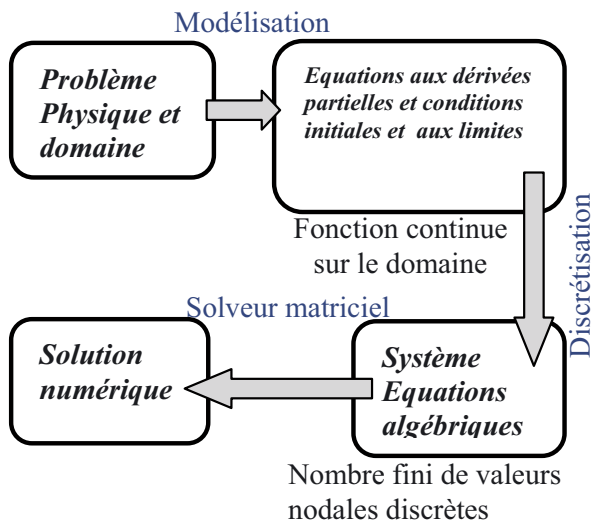

La simulation est réalisée avec un logiciel multiphysique du commerce $(\mathrm{COMSOL})^{1}$. Ce logiciel est basé sur la méthode des éléments-finis et la dynamique du moteur est résolue sur la base d'un maillage mobile.

Les résultats numériques présentés dans ce travail sont obtenus avec un maillage de 26486 éléments et une résolution temporelle lors de la simulation des cycles de $\Delta t=0,01 \mathrm{~s}$.

1 D'autres codes du commerce permettent également de simuler le présent problème (CFX, Fluent, Star-CD etc.). 


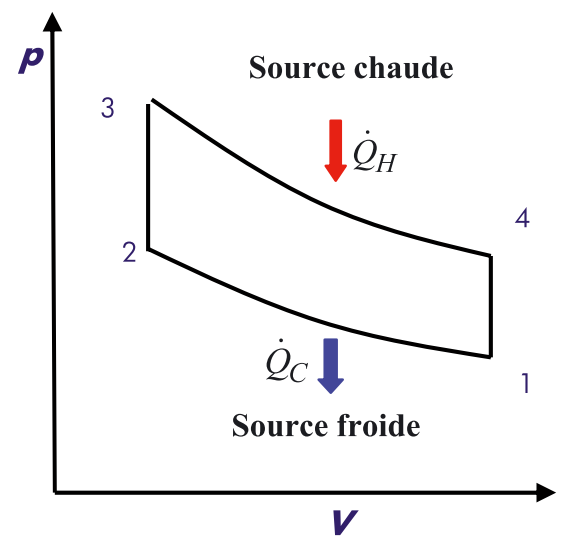

Fig. 2. Cycle du moteur Stirling dans un diagramme $p-V$; transformation entre les points $1-2$ : le gaz froid est comprimé par le piston moteur; transformation entre les points 2-3 : le piston déplaceur chasse le gaz vers la cellule chaude où il s'échauffe à volume constant; transformation entre les points $3-4$ : le gaz se détend en poussant le piston de travail ; c'est le temps moteur; transformation entre les points 4-1 : le piston chasse le gaz vers la source froide.

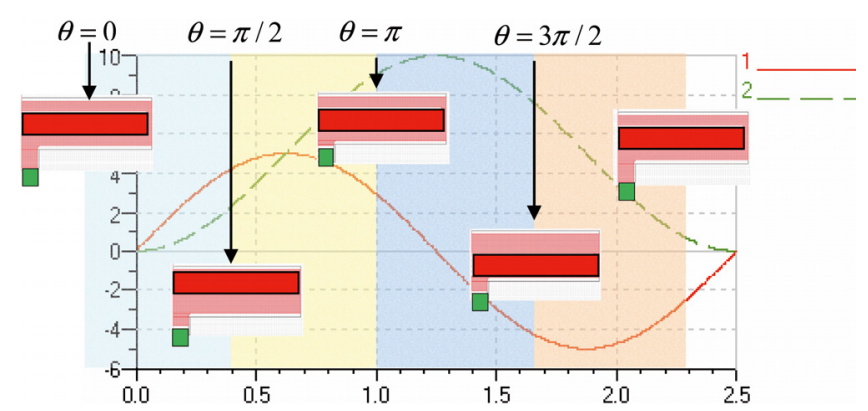

Fig. 3. Évolution des positions des pistons déplaceur (1) et moteur (2) en fonction du temps.

\section{Résultats numériques}

La simulation du cas du moteur de type gamma (prototype I) avec des températures imposées $T_{\mathrm{C}}$ et $T_{\mathrm{F}}$ de $301 \mathrm{~K}$ et $290 \mathrm{~K}$, respectivement, et dont les conditions de fonctionnement correspondent au déplacement du piston moteur et piston déplaceur représentés sur la figure 3.

Cette figure résume l'évolution des positions des pistons moteur et déplaceur ainsi que le schéma illustratif du moteur en quatre positions caractéristiques sur une période représentative. Les résultats numériques seront représentés en ces différents points et correspondent à :

- Point A : piston moteur au point mort bas, piston déplaceur à mi-course $(\theta=0)$.

- Point $\mathrm{B}$ : piston moteur à mi-course, piston déplaceur au point mort haut $(\theta=\pi / 2)$.

- Point $\mathrm{C}$ : piston moteur au point mort haut, piston déplaceur à mi-course $(\theta=\pi)$.

- Point D : piston moteur à mi-course, piston déplaceur au point mort bas $(\theta=3 \pi / 2)$.

Du point $\mathrm{A}$ au point $\mathrm{B}$ les deux pistons ont un mouvement ascensionnel. L'ascension du déplaceur chasse le fluide de la zone supérieure (au-dessus du déplaceur) vers la zone inférieure (voir Fig. 4a).

Les lignes de suivi de particule représentent bien ce déplacement d'air. Une accélération importante est observée (couleur rouge) au niveau du canal où le rétrécissement engendre un accroissement important des vitesses afin d'assurer la conservation de masse ${ }^{2}$.

Il est à signaler que l'étroitesse du passage ainsi que cet accroissement de vitesse donnera lieu à des gradients importants de vitesse et engendrera des cisaillements et pertes non négligeables. Une faible recirculation apparaît dans le cylindre vertical car le piston moteur est à proximité du point mort bas (vitesse très faible).

Le champ de température (Fig. 4b) illustre également ce passage de fluide de la zone supérieure froide vers la zone inférieure froide. L'intrusion du fluide chaud dans le domaine froid est très clairement illustrée par le dégradé de couleur. Ce champ de température met également en évidence l'importance des échanges au niveau de la paroi inférieure.

Le gaz chaud est refroidi sur une zone inférieure au $1 / 4$ du rayon.

La faiblesse des vitesses dans les zones supérieure et inférieure est illustrée clairement par les champs de pression (Fig. 4c) presque constants.

La zone supérieure est en compression alors que la zone inférieure est en cours de détente.

La puissance fournie par le moteur est directement donnée par le produit de la force qu'exerce le gaz sur les pistons par la vitesse de déplacement de ces derniers.

Les pertes du système concernent les différentes irréversibilités ainsi que le transfert de chaleur de la source chaude vers la source froide lors de cette transformation.

Afin de suivre les échanges, nous représentons (sur Fig. 5) les champs de température en différents points (A, B, C et D) représentant un cycle type lors du fonctionnement établi.

La figure 6 illustre l'alternance du déplacement du fluide entre les deux réservoirs, supérieur et inférieur. Cette alternance représente clairement les intrusions alternatives du fluide chaud et son refroidissement le long de la face froide (points A et B) ainsi que le réchauffement du fluide le long de la paroi chaude supérieure lors de la deuxième moitié du cycle (points $\mathrm{C}$ et $\mathrm{D}$ ).

L'utilisation d'un régénérateur plus efficace permettra de réduire cette perte et d'atténuer l'échange entre ces deux surfaces.

Le fluide chaud réchauffera la grille poreuse lors de son mouvement descendant (points A et B) et le fluide froid récupérera cette énergie (de la grille poreuse) lors de son mouvement ascendant ( $\mathrm{C}$ et $\mathrm{D})$.

Il est à signaler la forte irrégularité spatiale des transferts locaux d'énergie au vu de la complication de l'écoulement d'une part au niveau spatial et de l'instationnarité continuelle avec des inversions d'écoulement, d'autre part. Les champs de vitesse correspondant aux champs de température (Fig. 5) sont représentés sur la figure 6 .

\footnotetext{
2 à la compressibilité près.
} 


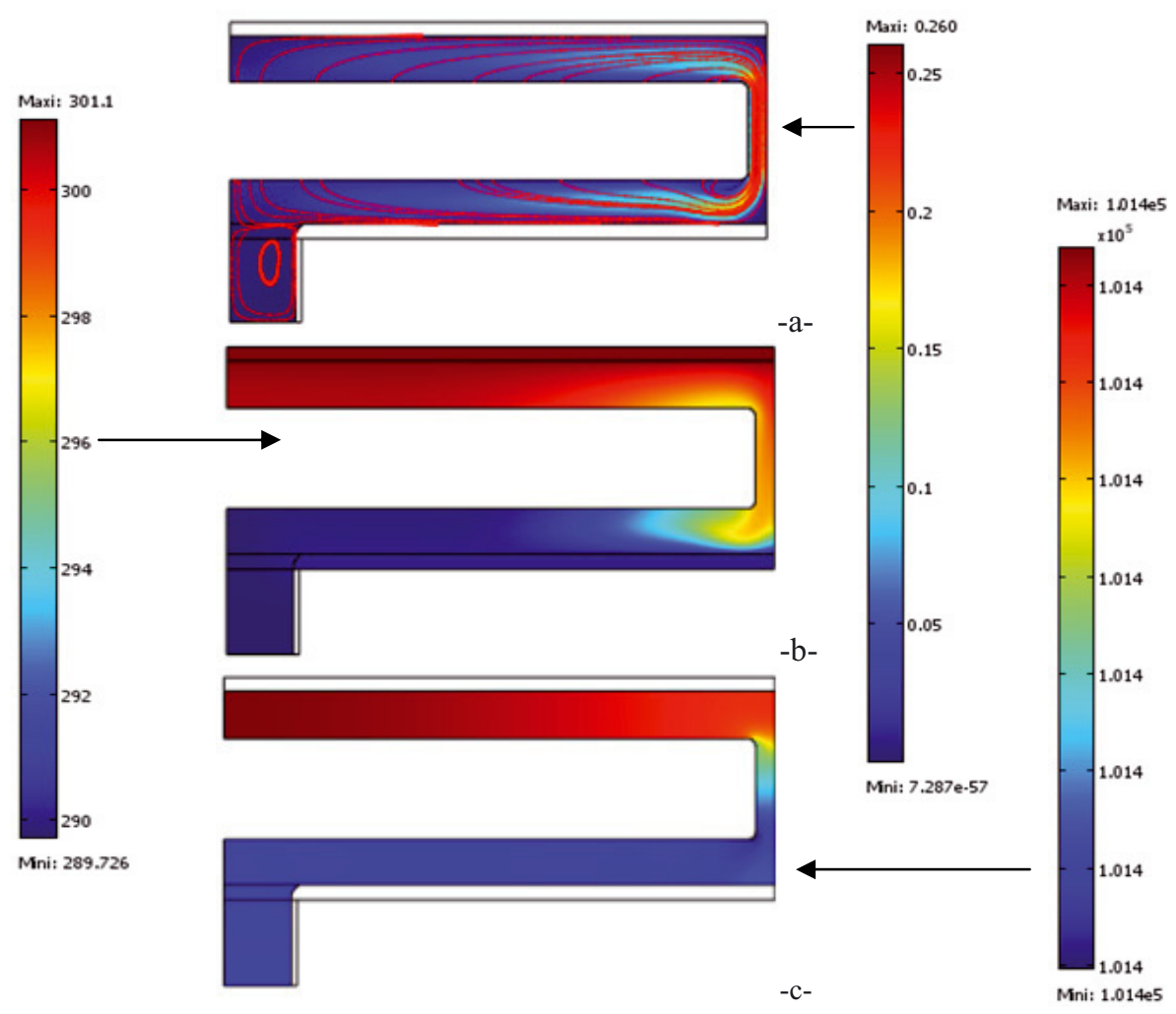

Fig. 4. Champs de vitesse (a), de température (b), et de pression (c), obtenue au point A (piston moteur au point mort bas, piston déplaceur à mi-course $\theta=0$ ).

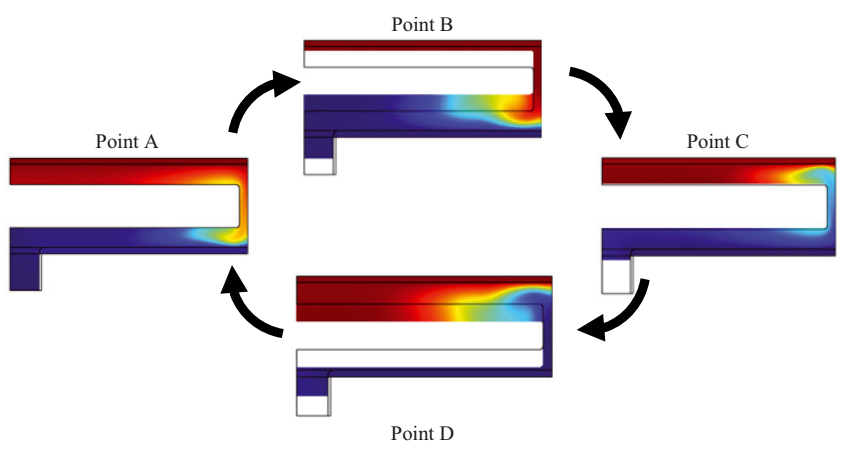

Fig. 5. Champs de température en différents points d'un cycle représentatif.

Cette figure illustre le caractère du jet issu de la fente qui relie les deux zones fluides. On remarque, de plus, l'apparition d'une recirculation importante (points B et D) en amont du jet, suivi par une recirculation en aval. Nous observons une équivalence dynamique entre les points B et D (A et C). Néanmoins, une différence sur la structure et l'intensité existe à cause de l'arrivée simultanée du fluide dans la zone inférieure du moteur (point B) à la fois de la fente et du piston moteur (mouvement ascensionnel). Cette différence accroît la compression. Le déplacement du piston moteur affecte de manière similaire la similitude entre les points A et $\mathrm{C}$.

Le déplacement du piston déplaceur engendre une surpression et dépression de part et d'autre.

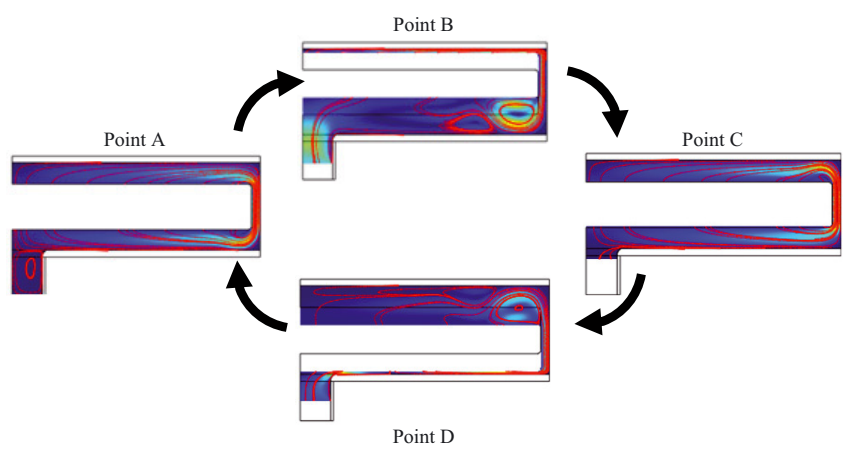

Fig. 6. Champs de vitesse en différents points d'un cycle représentatif.

La soudaine accélération au niveau de la fente est illustrée par la baisse en pression.

La compressibilité conséquente du chauffage et du refroidissement est moins visible car couplée aux phénomènes précédemment cités.

Une surpression (dépression) est visible à proximité immédiate du piston moteur point $\mathrm{C}(\mathrm{D})$.

Cette interaction fluide-structure (pression, surface) engendre une force au niveau du piston qui s'exprime en travail utile issu de cette transformation.

Le travail déterminé par la simulation numérique est comparé à celui obtenu expérimentalement sur le prototype gamma. 


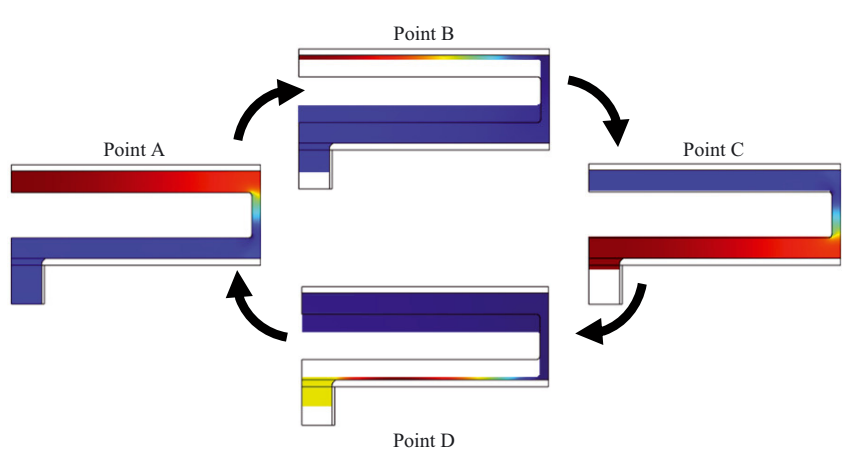

Fig. 7. Champs de pression en différents points d'un cycle représentatif.

Tableau 1. Comparaison du travail du moteur obtenu numériquement et expérimentalement.

\begin{tabular}{cc}
\hline Travail & Travail par cycle $[\mathrm{J}]$ \\
\hline Numérique & $8,02 \times 10^{-4}$ \\
Expérience & $10,2 \times 10^{-4}$ \\
\hline
\end{tabular}

Un accord acceptable est obtenu comme résumé sur le tableau 1. Néanmoins, une différence d'environ $20 \%$ est observée.

Cette différence est imputable, d'une part, aux erreurs des mesures expérimentales (au moins de l'ordre de $10 \%$ ) et à la difficulté de maintenir une température constante sur les faces du prototype (au vu de l'irrégularité des transferts de chaleur au cours du temps) et à proximité immédiate du rayon externe (sortie de fente), d'autre part. La seconde limitation est numérique car la simulation est effectuée en géométrie $2 \mathrm{D}$ et le caractère tridimensionnel du prototype est certain.

Une comparaison des cycles obtenus numérique et expérimental est présentée sur la figure 8.

\section{Conclusion}

Ce présent travail de modélisation et de simulation numérique a permis d'illustrer la progression du savoirfaire croissant d'une part et de l'accroissement de la puissance des ordinateurs d'autre part. Cette approche permet une exploration à moindre coût des différents paramètres de contrôles afin d'identifier les conditions et géométries optimales de fonctionnement des systèmes.

Ce présent travail appliqué à la modélisation et de simulation numérique des moteurs alternatifs de type Stirling, a permis d'établir les phénomènes dominants à prendre en compte afin de reproduire les phénomènes physiques qui contrôlent le cycle de transformations. La résolution numérique du modèle a permis d'obtenir des valeurs instantanées des grandeurs physiques locales en régime établi; la prédiction du cycle au cours du temps qui donne accès aux valeurs du travail ainsi que le rendement de la machine simulée.

Un très bon accord est constaté dans la comparaison des cycles $p$ - $V$ obtenus numériquement

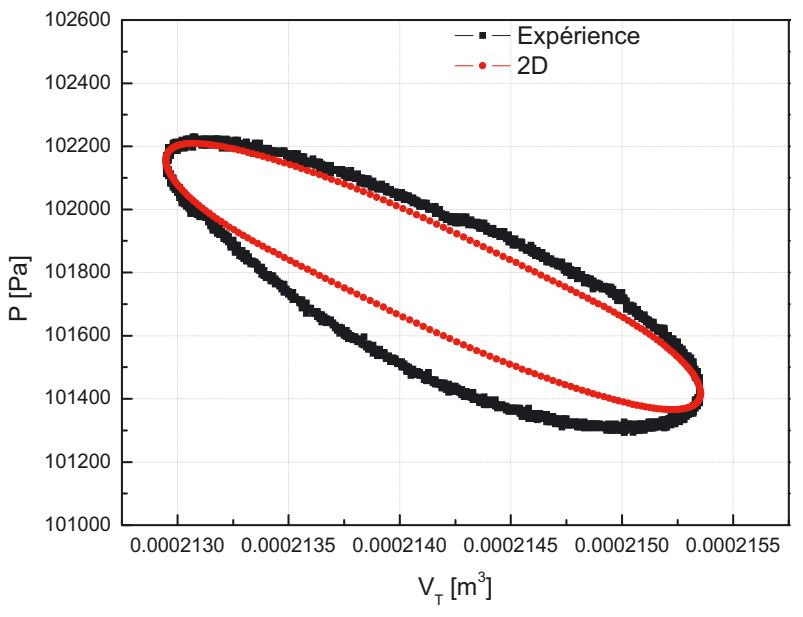

Fig. 8. Diagramme $p-V$ obtenu expérimentalement et numériquement.

et expérimentalement sur un prototype moteur de démonstration.

Par ailleurs les moteurs analysés constituent une solution pour la conversion efficace des énergies renouvelables et des chaleurs perdues en travail. Ils offrent un bon rendement de fonctionnement, tout en présentant une grande souplesse d'adaptation. La présente modélisation permettra une exploration des différents paramètres de contrôle afin d'identifier les conditions et géométries optimales (dimensions, matériaux, coefficient de transfert de chaleur, etc.) de fonctionnement.

\section{Annexe}

La modélisation de cette transformation (écoulement et transfert) du gaz enfermé dans le cylindre moteur est basée sur la résolution des équations de conservation qui s'expriment comme suit :

- équation de continuité de la masse :

$$
\frac{\partial \rho}{\partial t}+\nabla(\rho u)=0
$$

- équation de quantité de mouvement :

$$
\begin{aligned}
\rho \frac{\partial u}{\partial t}+\rho(u \nabla) u=\nabla[-p I & +\mu\left(\nabla u+(\nabla u)^{T}\right) \\
& \left.-\left(\frac{2 \mu}{3}-k\right)(\nabla u) I\right]
\end{aligned}
$$

- équation de l'énergie pour le solide (parois)

$$
\rho c_{\mathrm{p}} \frac{\partial T_{\mathrm{S}}}{\partial t}+\nabla\left(-\lambda \nabla T_{\mathrm{S}}\right)=0
$$

et pour le gaz de travail

$$
\rho c_{\mathrm{p}}\left(\frac{\partial T_{\mathrm{L}}}{\partial t}+(u \nabla) T_{\mathrm{L}}\right)=\nabla\left(\lambda \nabla T_{\mathrm{L}}\right)+\frac{D p}{D t}-(\tau: \nabla u)
$$


Le dernier terme de l'équation (4) représente la dissipation et on l'exprime comme :

$$
-(\tau: \nabla u)=\mu \phi_{u}
$$

où $\phi_{u}$ est la fonction de dissipation donnée en coordonnées cylindriques $(r, z)$ :

$$
\begin{aligned}
\phi_{u}= & 2\left[\left(\frac{\partial u_{r}}{\partial r}\right)^{2}+\left(\frac{u_{r}}{r}\right)^{2}+\left(\frac{\partial u_{z}}{\partial z}\right)^{2}\right] \\
& +\left[\frac{\partial u_{r}}{\partial z}+\frac{\partial u_{z}}{\partial r}\right]^{2}-\frac{2}{3}\left[\frac{1}{r} \frac{\partial}{\partial r}\left(r u_{r}\right)+\frac{\partial u_{z}}{\partial z}\right]^{2}
\end{aligned}
$$

En absence de ce terme $\left(\phi_{u}=0\right)$, toutes les formes d'énergie impliquées dans l'équation de l'énergie (cinétique, interne et potentielle) sont interchangeables. Le cas $\phi_{u}>0$, signifie qu'il y a dégradation d'énergie mécanique en chaleur. À ces équations, on associe l'équation d'état du fluide (gaz parfait $p=\rho r T, r=$ $287 \mathrm{~J} . \mathrm{kg}^{-1} \cdot \mathrm{K}^{-1}$ pour l'air).

Ces équations aux dérivées partielles forment un système de cinq équations non linéaires et couplées. Ce système est à l'heure actuelle impossible à résoudre analytiquement. Il est alors nécessaire de rechercher une solution approchée discrète par voie numérique.

La simulation est réalisée avec un logiciel multiphysique du commerce (COMSOL). Ce logiciel est basé sur la méthode des éléments finis et la dynamique du moteur est résolue sur la base d'un maillage mobile.

Les conditions aux limites appliquées aux frontières du domaine considéré (Fig. 1b) sont résumées ci-dessous.

- Les surfaces horizontales externes sont les plaques chaude et froide respectivement à $T_{\mathrm{C}}$ et $T_{\mathrm{F}}$.

- Le transfert entre ces plaques (internes) et le fluide sont données par : $n_{\mathrm{S}}\left(k_{\mathrm{S}} \nabla T_{\mathrm{S}}\right)+n_{\mathrm{f}}\left(k_{\mathrm{f}} \nabla T_{\mathrm{f}}\right)=0$.

- Le piston moteur (déplaceur) est considéré comme un bon isolant (adiabaticité) $n(-k \nabla T)=0$.

- Les vitesses du piston et du déplaceur sont données respectivement par $u=\partial z_{\mathrm{m}} / \partial t$ et $u=\partial z_{\mathrm{d}} / \partial t$.

- Concernant les frontières verticales du domaine considéré, on a une condition de symétrie sur l'axe central du moteur $(r=0)$ et sur la face verticale externe au fluide, la continuité des flux est donnée par $-n\left(-k \nabla T+\rho C_{\mathrm{p}} u T\right)=0$.

\section{Références}

[1] M. El Ganaoui, R. Bennacer, On some latent heat effects on the floating zone growth, Adv. Sp. Res. 36 (2005) 96-98

[2] O. Brisson, M. El Ganaoui, A. Simmonet, J.C. Launay, Experimental determination of physical parameters of $\mathrm{AgGaS}_{2}$ crystal and numerical and experimental analysis of the Bridgman-Stockbarger solidification for the growth of crystal in ampoule with conical schape, J. Cryst. Growth 204 (1999) 201-212

[3] K. Séfiane, R. Bennacer, M. El Ganaoui, Numerical investigation of the role of thermal evaporation profile initiating Marangoni convection in capillary tubes, Mécanique \& Industries 5 (2004) 575-581

[4] R. Bennacer, K. Sefiane, M. El-Ganaoui, C. Buffone, Numerical investigation of the role of the non-uniform evaporation rate in initiating Marangoni convection in capillary tubes, Int. J. Numer. Methods Heat Fluid Flow 14 (2004) 877-890

[5] A. Robson, Development of a computer model to simulate a low temperature differential Ringbom Stirling engine, Thermo- and GFD modelling of Stirling machines, 2005, pp. $350-357$

[6] S. Andersen, Numerical simulation of cyclic thermodynamic processes, thése soutenue en mars 2006

[7] R. Tan, K. Mahkamov, D. Djumanov, Experimental and numerical investigations on a small physical model of a solar Stirling engine, Thermo-and GFD modelling of Stirling machines, 2005, pp. 74-85

[8] N. Martaj, L. Grosu, P. Rochelle, Exergetical analysis and design optimization of the Stirling engine, Int. J. Exergy 3 (2006) 45-67

[9] G. Walker, Stirling-Cycle Machines, Clarendon Press Ed., 1973

[10] R. Senft, Ringbom Stirling Engines, Oxford University Press, 1993, ISBN 0-19-507798-9

[11] N. Kagawa, Regenerative Thermal Machines (Stirling and Vuilleumier Cycle Machines) for Heating and Cooling, Int. Institute of Refrigeration ed., 2000, ISBN 291314905-7

[12] A.J. Organ, The Regenerator and the Stirling Engine, Mech. Eng. Pub. Ltd ed., 1997, ISBN 1860580106

[13] www.stirlingengine.co.uk

[14] www.photologie.net 